\title{
New data on the millipede genus Altajosoma Gulička, 1972 from southwestern Siberia, Russia (Diplopoda: Chordeumatida: Diplomaragnidae)
}

\section{Новые сведения о двупарноногих многоножках рода Altajosoma Gulička, 1972 с юга Западной Сибири, Россия (Diplopoda: Chordeumatida: Diplomaragnidae)}

\author{
P.S. Nefediev ${ }^{1,2}$ \\ П.С. Нефедьев ${ }^{1,2}$
}

\footnotetext{
${ }^{1}$ Altai State University, Lenin Avenue, 61, Barnaul656049 Russia. E-mail: p.nefediev@mail.ru

2 Tomsk State University, Lenin Avenue, 36, Tomsk 634050 Russia.

${ }^{1}$ Алтайский государственный университет, проспект Ленина, 61, Барнаул 656049 Россия.

2 Томский государственный университет, проспект Ленина, 36, Томск 634050 Россия.
}

KEY WORDS: Diplopoda, Diplomaragnidae, Altajosoma, taxonomy, fauna, new records, Siberia, Russia.

КЛЮЧЕВЫЕ СЛОВА: Diplopoda, Diplomaragnidae, Altajosoma, таксономия, фауна, новые находки, Сибирь, Россия.

ABSTRACT. Based on fresh and re-examined material from SW Siberia, the distributions of seven diplomaragnid millipede species have been supplemented, refined and updated. The following few taxonomic changes are proposed: Altajosoma bakurovi (Shear, 1990), stat. revalid. ex A. bakurovi bakurovi (Shear, 1990), Altajosoma bakurovi longiprocessum Mikhaljova, $2000=A$. longiprocessum Mikhaljova, 2000, stat.n. A. bakurovi (Shear, 1990) is recorded from the Republic of Khakassia for the first time, A. corniferum Mikhaljova, Nefediev et Nefedieva, 2008 is new to the Altai Province. A description of the posterior gonopods of $A$. deplanatum (Stuxberg, 1876), as well as the diagnosis of the genus in general are supplemented. The distributions of all Altajosoma species encountered are mapped.

How to cite this article: Nefediev P.S. 2019. New data on the millipede genus Altajosoma Gulička, 1972 from southwestern Siberia, Russia (Diplopoda: Chordeumatida: Diplomaragnidae) // Arthropoda Selecta. Vol.28. No.2. P.206-212. doi: 10.15298/arthsel. 28.2.03

РЕЗЮМЕ. По новому и повторно изученному коллекционному материалу с юга Западной Сибири дополнены, уточнены и обновлены области распространения для семи видов диплопод-нитеносцев. Предложены несколько таксономических изменений: Altajosoma bakurovi (Shear, 1990), stat. revalid. ex A. bakurovi bakurovi (Shear, 1990), Altajosoma bakurovi longiprocessum Mikhaljova, 2000 = A. longiprocessum Mikhaljova, 2000, stat.n. A. bakurovi (Shear, 1990) впервые отмечен в Респуб- лике Хакасия, $A$. corniferum Mikhaljova, Nefediev et Nefedieva, 2008 оказался новым для Алтайского края. Дополнено описание задних гоноподов $A$. deplanatum (Stuxberg, 1876), а также диагноз рода в целом. Для всех видов Altajosoma выполнено картирование ареалов.

\section{Introduction}

The millipede genus Altajosoma Gulička, 1972 is currently known to be represented by 12 species, which mainly occur in the south of western and central Siberia, as well as Eastern Kazakhstan [Mikhaljova, 2017, 2019]. Only one species, A. golovatchi Shear, 1990, which inhabits the southern part of the Krasnoyarsk Province, penetrates beyond the Urals and shows remarkable disjunct outposts in the southern Urals and Cis-Ural region.

The type material of both subspecies of A. bakurovi (Shear, 1990), now deposited in the collections of the Zoological Museum of the Lomonosov Moscow State University, Moscow (ZMUM) and the Federal Scientific Center of the East Asia Terrestrial Biodiversity, Far Eastern Branch of the Russian Academy of Sciences, Vladivostok (FSCB), both Russia, has been re-examined.

SEM micrograph was prepared at the Laboratory of Phylogeny and Faunogenesis, Institute of Systematics and Ecology of Animals, Siberian Branch of the Russian Academy of Sciences, Novosibirsk, Russia (ISEA), using a Hitachi TM-1000 scanning electron microscope. The distribution maps were composed using QGIS 3.4.4.

The material treated herein has been deposited in the collection of the Altai State University, Barnaul, Russia (ASU). Literature references to the species concern their distribution areas. s.l. — same locality; p.t. — pitfall traps. 




Fig. 1. Distribution of Altajosoma species: bakurovi (Shear, 1990) (circle) and katunicum Mikhaljova, 2000 (triangle). Previously known localities marked in black, new records given in white.

Рис. 1. Распространение видов Altajosoma: bakurovi (Shear, 1990) (круг) и katunicum Mikhaljova, 2000 (треугольник). Черным отмечены ранее известные места находок, новые находки отмечены белым.

Taxonomic part

\section{Class Diplopoda}

Family DIPLOMARAGNIDAE

Altajosoma bakurovi (Shear, 1990) Fig. 1.

Diplomaragna bakurovi Shear, 1990: 22, 23: figs. Diplomaragna bakurovi — Mikhaljova, 1993: 18; Nefediev, Nefedieva, 2008a: 117

Altajosoma bakurovi - Mikhaljova, 2000: 161, figs; Mikhaljova, Golovatch, 2001: 108; Nefediev, 2002a: 30; Mikhaljova Nefediev, 2003: 86; Nefediev, Nefedieva, 2005: 177.

Altajosoma bakurovi bakurovi - Mikhaljova, 2000: 161, figs; 2004: 178, figs, 116: map; 2017: 218, figs, 219: map; Mikhaljova et al., 2008: 51; Nefediev, Nefedieva, 2006: 98; 2007b: 161; 2007c 99; 2008b: 62; 2011: 100; 2012a: 47; 2012b: 51; 2013: 87; 2017 294; Nefedieva, Nefediev, 2008: 123; Nefedieva et al., 2014: 65; 2015: 147

non Altajosoma bakurovi bakurovi - Nefediev, Nefedieva, 2007a: 139.

MATERIAL EXAMINED, 5 juv., Russia, Tomsk Area, Tomsk District, near Zorkaltsevo, 56.521326 ${ }^{\circ} \mathrm{N}, 84.733903^{\circ} \mathrm{E}$, Pinus sibirica forest, soil samples, 24.VII.2001; 1 juv., s.1., soil samples, 24.VIII.2001; 9 juv., same Area and District, near Kislovka, 56. $430654^{\circ} \mathrm{N}, 84.900971^{\circ} \mathrm{E}$, Populus tremula forest, soil samples,
10-11.VI.2001; 1 ○', 1 ㅇ, 5 juv., s.1., soil samples, 18-22.VII.2001; 6 ○ $^{\top}, 12$ Oᄋ, 1 fragm., s.1., p.t., 6-26.VIII.2001; $3 \sigma^{\top} \sigma^{\top}, 1$ ㅇ, 1 juv., s.1., soil samples, 26.VIII.2001; $41 \sigma^{\top} \sigma^{7}, 23$ oᄋ, 3 fragm., s.1., p.t., 26.VIII.-18.IX.2001; 4 juv., s.1., 9.X.2001, all leg. P.S. Nefediev, J.S. Nefedieva; $1 \sigma^{7}$, Russia, Republic of Khakassia, Altai

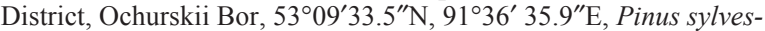
tris forest, 30.IX.2008, leg. D.I. Pogrebnyak; $2 \Im^{\top} \sigma^{\top}, 3$ 우, 1 juv., Russia, Republic of Altai, Ongudai District, old Chike-Taman Pass, $50.642909^{\circ} \mathrm{N}, 86.311878^{\circ} \mathrm{E}$, Caragana thicket, $1275 \mathrm{~m}$ a.s.1., 5.VIII.2007, leg. P.S. Nefediev, J.S. Nefedieva; 1 ऽ, 1 , same Republic, Turochak District, Altai State Nature Reserve, NW of Lake Teletskoye, cordon Baigazan, $51^{\circ} 45^{\prime} 34.1^{\prime \prime} \mathrm{N}, 87^{\circ} 26^{\prime} 03.6^{\prime \prime} \mathrm{E}$, Padus avium forest, $490 \mathrm{~m}$ a.s.1., 1.X.2013; $2 \mathrm{\sigma}^{\top} \sigma^{7}, 1$, , near s.1., $51^{\circ} 45^{\prime} 32.7^{\prime \prime} \mathrm{N}, 87^{\circ} 26^{\prime} 05.8^{\prime \prime} \mathrm{E}$, Padus avium forest, ca $480 \mathrm{~m}$ a.s.l., 1.X.2013, all leg. M.B. Sakhnevich; 1 ऽ", same Republic, Ulagan District, Aigulakskii Mt. Range, near source of Sardyma River, $50^{\circ} 24^{\prime} \mathrm{N}, 87^{\circ} 33^{\prime} \mathrm{E}$, rocky alpine meadow with rock screes, 2400$2500 \mathrm{~m}$ a.s.1., 29.VII.2016, leg. A.A. Fomichev; 1 \% , 1 ㅇ, 1 subadult $\sigma^{\prime}$, 1 juv., near s.1., $50^{\circ} 24^{\prime} \mathrm{N}, 87^{\circ} 34^{\prime} \mathrm{E}$, alpine meadow, under logs, $1880 \mathrm{~m}$ a.s.1., 29.VII.2016, leg. Yu.V. Dyachkov; 1 フ', same Republic, same District, Kuraiskii Mt. Range, watershed of Korumduairy River and Yarlyamry River, $50^{\circ} 19^{\prime} \mathrm{N}, 87^{\circ} 42^{\prime} \mathrm{E}$, Larix sibirica forest, moss rocky slope, 2000-2150 m a.s.1., 7.VIII.2016, leg. A.A. Fomichev; $5 \sigma^{7} \sigma^{\top}, 4$ 90,1 juv., same Republic, UstKoksa District, $0.5 \mathrm{~km} \mathrm{~W}$ of Ust-Koksa, $50^{\circ} 16^{\prime} 27.74^{\prime \prime} \mathrm{N}, 85^{\circ} 35^{\prime}$ 29.21"E, Betula forest, under stones, $980 \mathrm{~m}$ a.s.1., 14-15.VIII.2016, leg. Yu.V. Dyachkov; $3 \sigma^{7} \sigma^{7}, 1$, same Republic, Ongudai District, 7.5 air-km ENE of Seminskii Pass, Seminskii Mt. Range, environs of Mt Sarlyk, $51.06257^{\circ} \mathrm{N}, 85.70872^{\circ} \mathrm{E}$, alpine meadow with sparse Larix sibirica, $1970 \mathrm{~m}$ a.s.1., 21.VII.2018, leg. P.S Nefediev; $1 \sigma^{7}, 2$ juv., near s.1., $51^{\circ} 03.964^{\prime} \mathrm{N}, 85^{\circ} 42.421^{\prime} \mathrm{E}$, forest 
patch with Pinus sibirica and Picea obovata, small gaps, rocky, Betula rotundifolia, ca $1990 \mathrm{~m}$ a.s.l., sifting leaf litter and moss over 70 m, 21.VII.2018, leg. V.I. Gusarov, P.S. Nefediev; 8 juv., same Republic and District, 8 air-km ENE of Seminskii Pass, Seminskii Mt. Range, environs of Mt Sarlyk, $51^{\circ} 04.031^{\prime} \mathrm{N}, 85^{\circ} 42$. $779^{\prime}$ E, alpine meadow with Betula rotundifolia and Salix thicket, sparse trees of Pinus sibirica, Picea obovata and Larix sibirica, ca $2065 \mathrm{~m}$ a.s.1., sifting leaf litter and moss over $70 \mathrm{~m}, 21$. VII.2018, leg. V.I. Gusarov, M.F. Maurstad, V. Løveng; $6 \bigcirc^{\top} \sigma^{\top}, 2$ 우, 1 subadult $\sigma^{7}$, same Republic and District, 10 air-km NE of Seminskii Pass, Seminskii Mt. Range, summit of Mt Sarlyk, $51.077217^{\circ} \mathrm{N}$, $85.735567^{\circ} \mathrm{E}$, rocky desert with patches of mountain tundra, under stones, $2505 \mathrm{~m}$ a.s.1., 21.VII.2018; $9 \sigma^{\top} \sigma^{\top}, 2$ 우, near s.1., E slope of Mt Sarlyk, $51.0774112^{\circ} \mathrm{N}, 85.738094^{\circ} \mathrm{E}$, mountain tundra with rocky screes, under stones, $2400 \mathrm{~m}$ a.s.1., 21.VII.2018; $1 \mathrm{O}^{7}, 2$ 우, same Republic and District, 10.5 air-km NE of Seminskii Pass, Seminskii Mt. Range, foot of Mt Sarlyk, Lakes Tuyukskiye, 51. $075783^{\circ} \mathrm{N}, 85.742667^{\circ} \mathrm{E}$, mountain tundra, under stones, ca 2230 $\mathrm{m}$ a.s.1., 21.VII.2018; $1 \sigma^{\mathrm{T}}, 1$ \%, same Republic and District, 20 air$\mathrm{km} \mathrm{W}$ of Chibit, valley of Chuya River, near Shirlak Waterfall, right bank of Chuya River, $50.34358^{\circ} \mathrm{N}, 87.22225^{\circ} \mathrm{E}$, Betula forest with Larix sibirica, Caragana, small grass vegetation and green mosses, ca $1015 \mathrm{~m}$ a.s.1., 23.VII.2018; $3 \sigma^{\top} \sigma^{\top}, 2$ 우, same Republic and District, 20 air-km W of Chibit, valley of Chuya River, environs of Shirlak Waterfall, along Tektu River down the waterfall, $50.34542^{\circ} \mathrm{N}, 87.21915^{\circ} \mathrm{E}$, Betula forest with Alnus, Lonicera and tall grass vegetation, ca $1080 \mathrm{~m}$ a.s.1., 23.VII.2018, all leg. P.S. Nefediev; $2 \sigma^{\top} \sigma^{\top}, 1$ \%, near s.1., $50^{\circ} 20.690^{\prime} \mathrm{N}, 87^{\circ} 13.213^{\prime} \mathrm{E}$, Betula forest along Tektu River, sifting leaf litter over $40 \mathrm{~m}$, ca $1080 \mathrm{~m}$ a.s.1., 23.VII.2018, leg. V.I. Gusarov, M.F. Maurstad, V. Løveng.

DISTRIBUTION. This species is rather widespread in southwestern Siberia (E districts of the Novosibirsk Area, Altai Province and Republic of Khakassia, S districts of the Kemerovo and Tomsk areas, widely in the Republic of Altai) and south of central Siberia ( $\mathrm{S}$ districts of the Krasnoyarsk Province) (Fig. 1).

REMARKS. Originally described in Diplomaragna Attems, 1907 from the Novosibirsk Area (see Shear [1990]), this species was later transferred to Altajosoma, with the designation of the nominotypical subspecies, Altajosoma bakurovi bakurovi, whereas the second subspecies, A. $b$. longiprocessum Mikhaljova, 2000, was found in the Sayano-Shushenskii Nature Reserve, $\mathrm{S}$ part of the Krasnoyarsk Province (see Mikhaljova [2000]). The A. b. bakurovi-A. b. longiprocessum pair of subspecies remains the sole in Altajosoma. A restudy of the type material of both subspecies shows that they actually belong to different species. Altajosoma bakurovi is new to be reported from the Republic of Khakassia, as well as from its southern range in the Republic of Altai.

\section{Altajosoma baltyrgan Mikhaljova, 2013} Fig. 2.

Altajosoma katunicum pro parte - Mikhaljova et al., 2008 52 Nefedieva, Nefediev, 2008: 123; Nefediev, Nefedieva, 2013: 87; Nefedieva et al., 2014: 65; 2015: 149.

Altajosoma baltyrgan Mikhaljova, 2013: 2, 3-4, 6: figs.

NEW MATERIAL EXAMINED. $1 \sigma^{7}, 1$, Russia, Republic of Altai, Turochak District, Altai State Nature Reserve, NW of Lake Teletskoye, cordon Baigazan, 51 $45^{\prime} 35.0^{\prime \prime} \mathrm{N}, 87^{\circ} 26^{\prime} 02.3^{\prime \prime} \mathrm{E}$, Salix caprea forest, ca $480 \mathrm{~m}$ a.s.1., 30.IX.2013; $1 \sigma^{7}$, near s.1., $51^{\circ}$ $45^{\prime} 35.0^{\prime \prime} \mathrm{N}, 87^{\circ} 25^{\prime} 42.2^{\prime \prime} \mathrm{E}$, Padus avium forest, ca $470 \mathrm{~m}$ a.s.l., 3.X.2013, all leg. M.B. Sakhnevich.

MATERIAL RE-EXAMINED (specimens previously identified as A. katunicum Mikhaljova, 2000 and published by Mikhaljova et al. [2008]). 1 ऽ', Russia, Republic of Altai, Ulagan District, Altai State Nature Reserve, S of Lake Teletskoye, cordon Chiri, mouth of Chiri River, $51.363121^{\circ} \mathrm{N}, 87.835473^{\circ} \mathrm{E}$, Betula pendula forest, $465 \mathrm{~m}$ a.s.1., 11-12.VIII.2005; $1 \mathrm{\sigma ^{7 }}$, same Republic, Tu- rochak District, Altai State Nature Reserve, N of Lake Teletskoye, Yailyu, $51.769912^{\circ} \mathrm{N}, 87.617391^{\circ} \mathrm{E}$, Pinus sylvestris forest with Betula pendula, broad gully, $460 \mathrm{~m}$ a.s.1., 19.VIII.2005, all leg. P.S. Nefediev, J.S. Nefedieva.

DISTRIBUTION. This species has previously been known only from its type locality (near Mt Baltyrgan, Choya District, Republic of Altai). Now it is recorded from the Altai State Nature Reserve in the environs of Lake Teletskoye (Fig. 2).

REMARKS. A restudy of two $\sigma^{\top} \sigma^{7}$ from near cordon Chiri and at Yailyu in the environs of Lake Teletskoye, previously identified as A. katunicum by Mikhaljova et al. [2008], shows that both of them actually belong to $A$. baltyrgan. This species is recorded from outside its terra typica for the first time.

\section{Altajosoma corniferum Mikhaljova, Nefediev et Nefedieva, 2008 \\ Fig. 3.}

Altajosoma corniferum Mikhaljova, Nefediev et Nefedieva, 2008: 49, 50: figs.

Altajosoma corniferum - Nefediev, Nefedieva, 2008: 62.

MATERIAL EXAMINED. $2 \sigma^{\top} \sigma^{\top}$, Russia, Altai Province, Krasnoshchiokovo District, Tigirek State Nature Reserve, buffer zone, $51.180883^{\circ} \mathrm{N}, 82.973475^{\circ} \mathrm{E}$, Salix thicket, ca $800 \mathrm{~m}$ a.s.1., hand sampling, 17.VII.2014; $4 \sigma^{\top} \sigma^{\top}, 1$, same Province and District, Tigirek State Nature Reserve, Khankhara Site, ca 6 km NW of Tigirek, $51.184260^{\circ} \mathrm{N}, 82.973277^{\circ} \mathrm{E}$, Betula pendula and Salix forest, 675-815 $\mathrm{m}$ a.s.1., soil samples, 18.VII.2014, all leg. Yu.V. Dyachkov; $1 \sigma^{7}$, same Province, Zmeinogorsk District, near former Beloretsk, Tigirek State Nature Reserve, Beloretsk Site, left bank of Belaya River, right bank of Glukharikha River near its mouth, middle part of $\mathrm{W}$ slope, $51^{\circ} 00^{\prime} 15.31^{\prime \prime} \mathrm{N}, 82^{\circ} 45^{\prime} 47.04^{\prime \prime} \mathrm{E}$, glade with tall grass vegetation in chern taiga, $540 \mathrm{~m}$ a.s.1., p.t., 2028.VIII.2014; $2 \sigma^{7} \sigma^{7}$, near same locality, lower part of W slope, $51^{\circ} 00^{\prime} 14.00^{\prime \prime} \mathrm{N}, 82^{\circ} 45^{\prime} 45.00^{\prime \prime} \mathrm{E}$, chern taiga, ca $510 \mathrm{~m}$ a.s.l., p.t., 20-28.VIII.2014; $1 \sigma^{7}$, near same locality, upper and middle parts of W slope, $51^{\circ} 00^{\prime} 13.60^{\prime \prime} \mathrm{N}, 82^{\circ} 45^{\prime} 47.80^{\prime \prime} \mathrm{E}$, chern taiga, $550 \mathrm{~m}$ a.s.1., p.t., 20-28.VIII.2014; $1 \sigma^{\top}$, same Province, Krasnoshchiokovo District, near Tigirek, Tigirek State Nature Reserve, buffer zone, left bank of Malyi Tigirek River, near mouth of Voskresenka Channel, bottom of river valley, $51^{\circ} 08^{\prime} 30.50^{\prime \prime} \mathrm{N}, 83^{\circ} 02^{\prime} 43.40^{\prime \prime} \mathrm{E}$, Betula pendula forest, $500 \mathrm{~m}$ a.s.1., p.t., 29.VIII.-8.IX.2014; 1 ㅇ, same Province and District, near Tigirek, Tigirek State Nature Reserve, buffer zone, right bank of Malyi Tigirek River, bottom of river valley, $51^{\circ} 08^{\prime} 45.10^{\prime \prime} \mathrm{N}, 83^{\circ} 02^{\prime} 34.00^{\prime \prime} \mathrm{E}$, Betula pendula forest, $480 \mathrm{~m}$ a.s.1., p.t., 29.VIII.-8.IX.2014, all leg. T.M. Krugova, O.P. Antonova; $2 \sigma^{\top} \sigma^{\top}, 1$, same Province and District, Tigirek State Nature Reserve, buffer zone, Dragunskii Brook, $51.177247^{\circ} \mathrm{N}$, $82.970800^{\circ} \mathrm{E}$, Betula pendula forest with Salix and Ribes nigrum, $770 \mathrm{~m}$ a.s.1., hand sampling, in litter, 17-18.VIII.2016, leg. P.S. Nefediev, J.S. Nefedieva; $1 \sigma^{\gamma}, 1$, 1 subadult $\sigma^{7}$, same Province and District, Tigirek State Nature Reserve, Khankhara Site, top of $\mathrm{N}$ slope, $51.189621^{\circ} \mathrm{N}, 82.953090^{\circ} \mathrm{E}$, Betula pendula, Larix sibirica and Abies sibirica forest with Sorbus sibirica, $925 \mathrm{~m}$ a.s.1., hand sampling, in litter, 18.VIII.2016, leg. P.S. Nefediev; $1 \sigma^{7}, 1$, same Province and District, Tigirek State Nature Reserve, Khankhara Site, watershed of Khankhara River and Dragunskii Brook, $51^{\circ} 11^{\prime}$ $31.75^{\prime \prime} \mathrm{N}, 82^{\circ} 58^{\prime} 22.79^{\prime \prime} \mathrm{E}$, Betula pendula and Larix sibirica forest with Rubus idaeus, Salix caprea, Ribes spicatum, upper and middle parts of $\mathrm{N}$ slope, $850 \mathrm{~m}$ a.s.1., soil samples, 18.VIII.2016, leg. T.M. Krugova, L.Yu. Gruntova, V.V. Zelenskii, K.V. Smirnova, A.E. Pupkova, M.N. Terioshkina, R.V. Shcherbakova.

DISTRIBUTION. This species has previously been known only from its terra typica (Seminskii Pass, Ongudai District, Republic of Altai), presently being reported from the $\mathrm{S}$ districts of the Altai Province in the Tigirek State Nature Reserve (Fig. 3).

REMARKS. This species is recorded from outside its type locality for the first time. 


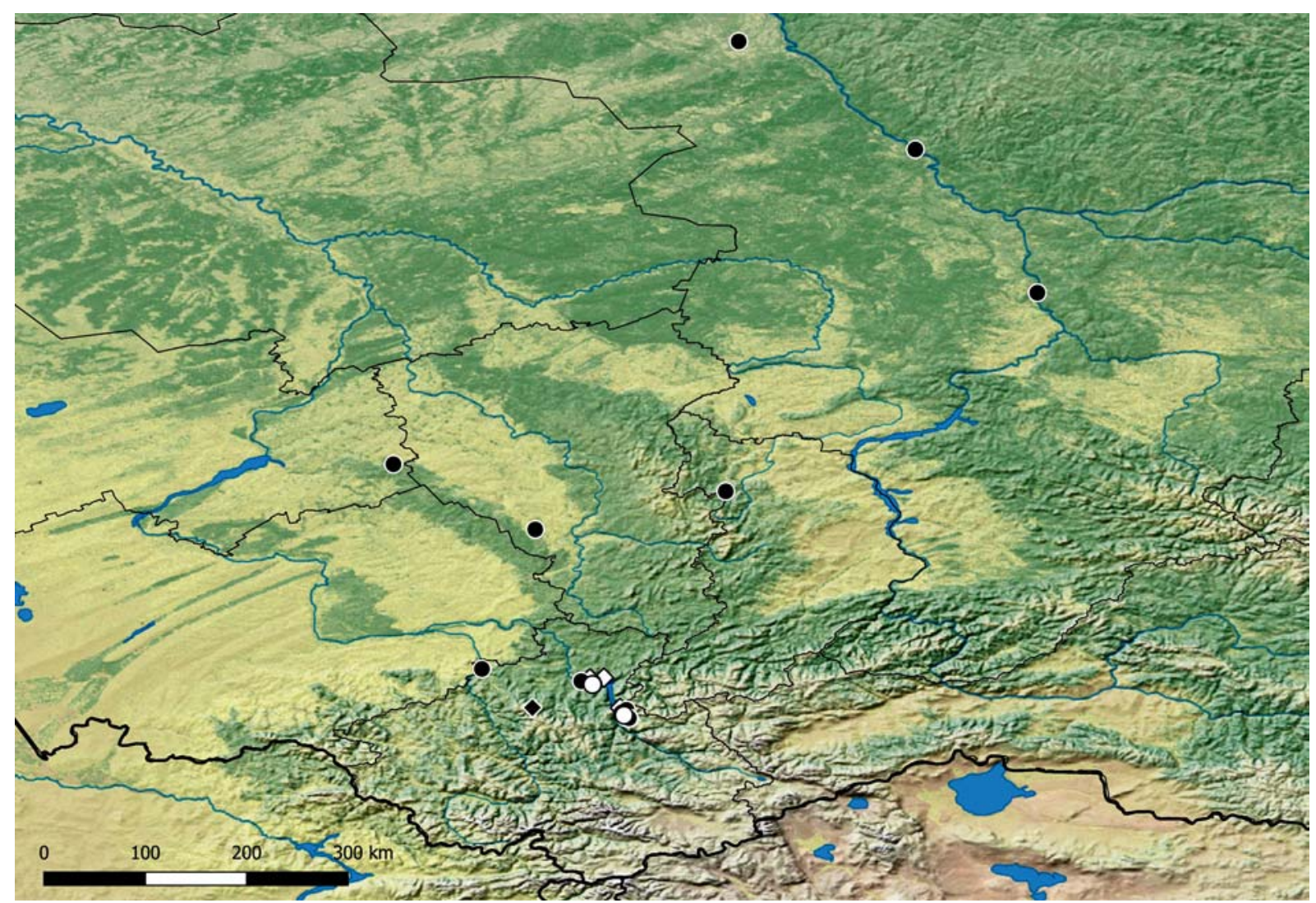

Fig. 2. Distribution of Altajosoma species: baltyrgan Mikhaljova, 2013 (diamond) and kemerovo (Shear, 1990) (circle). Previously known localities marked in black, new records given in white.

Рис. 2. Распространение видов Altajosoma: baltyrgan Mikhaljova, 2013 (ромб) и kemerovo (Shear, 1990) (круг). Черным отмечены ранее известные места находок, новые находки отмечены белым.

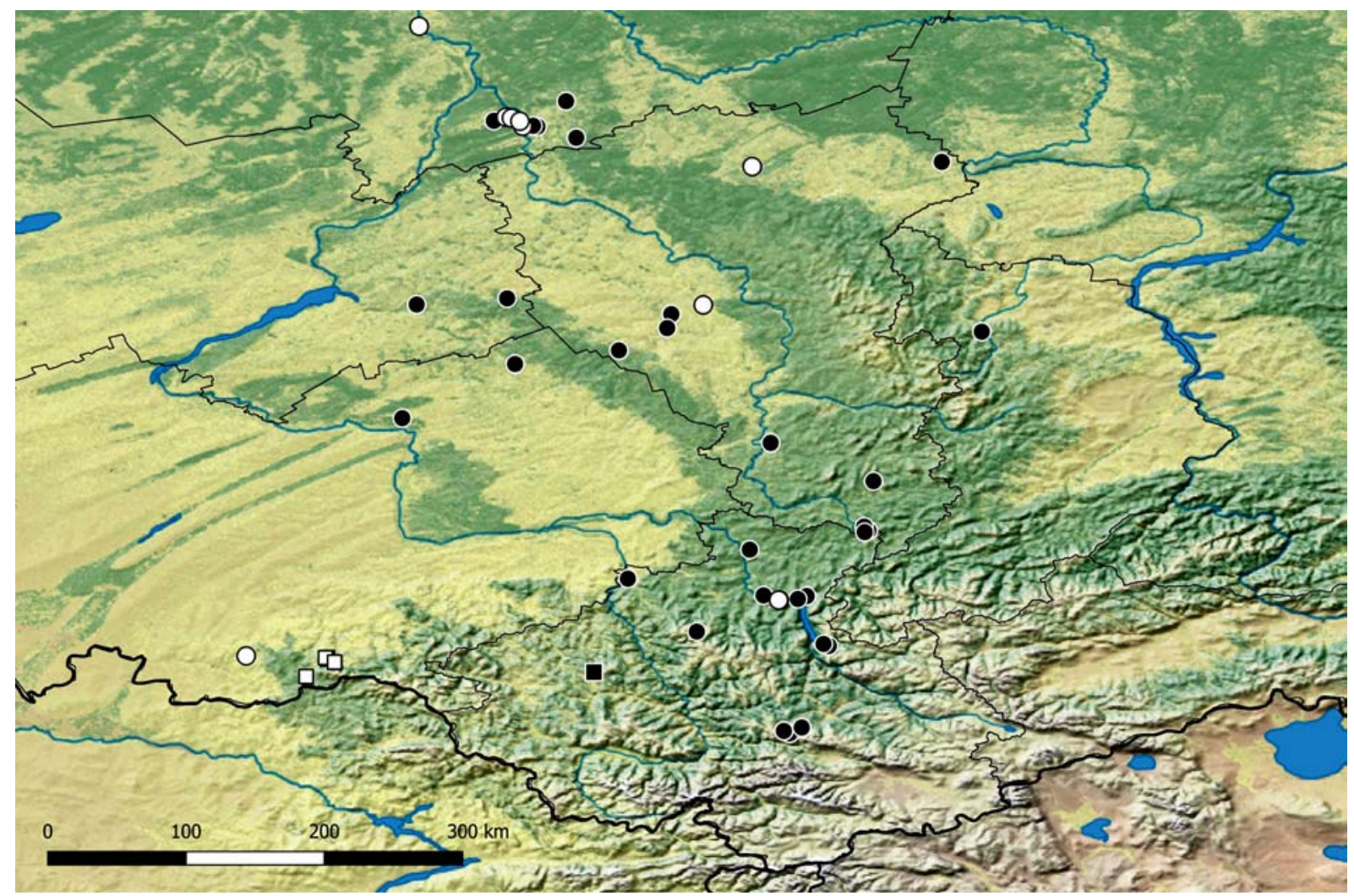



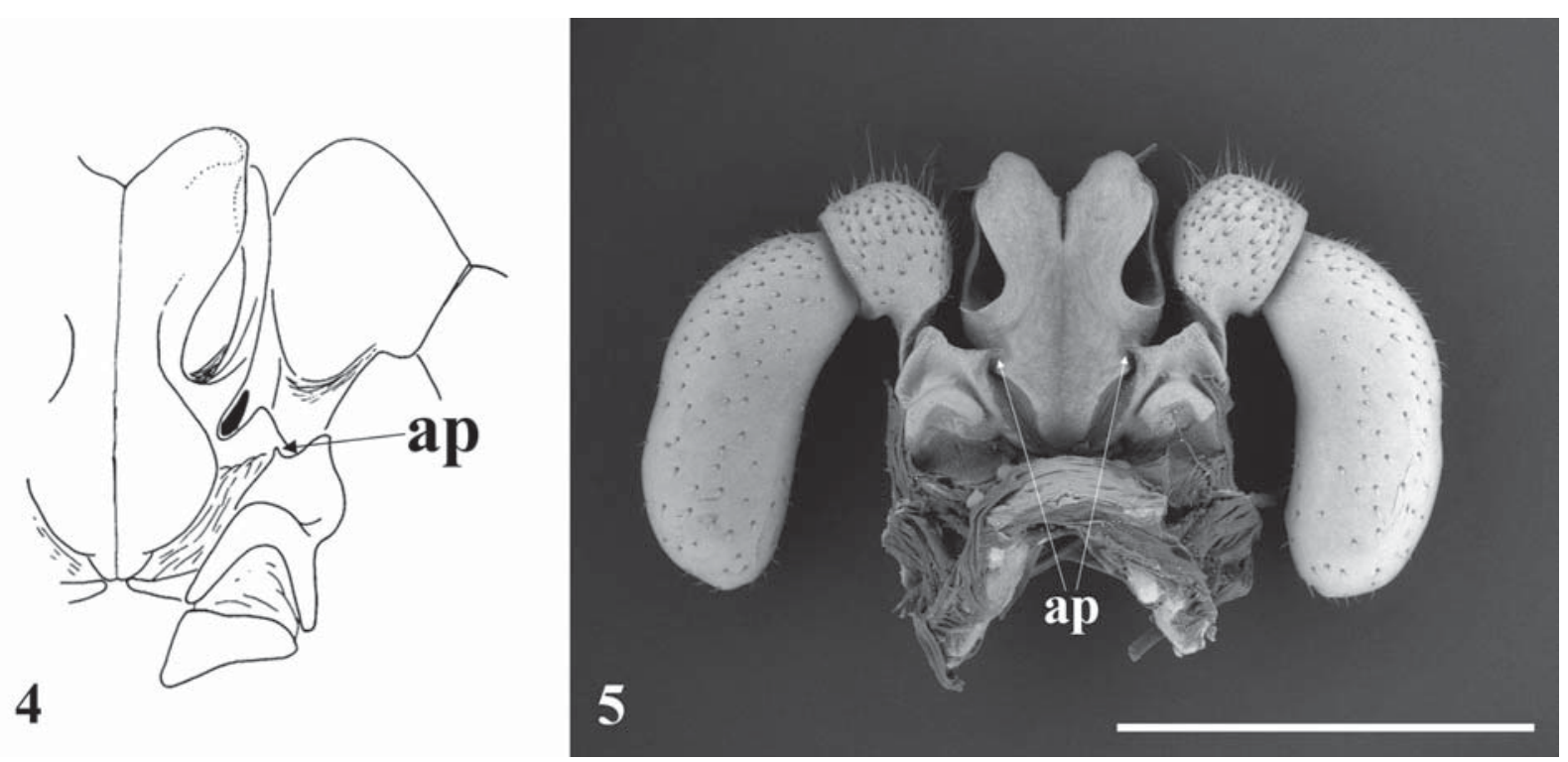

Figs 4-5. Altajosoma deplanatum (Stuxberg, 1876), male. 4, 5 - gonopods, oral view (after Shear [1990]), with some changes, without scale (4)); Republic of Altai, near cordon Baigazan (5). Scale bar: $1 \mathrm{~mm}$. Designations explained in text.

Рис. 4-5. Altajosoma deplanatum (Stuxberg, 1876), самец. 4, 5 - гоноподы, вид спереди (по Shear [1990] с некоторыми изменениями, без масштаба (4)); Республика Алтай, около кордона Байгазан (5). Масштаб: 1 мм. Объяснение обозначений дано в тексте.

\section{Altajosoma deplanatum (Stuxberg, 1876)} Fig. 3-5.

Craspedosoma deplanatum Stuxberg, 1876a: 36, figs. Craspedosoma deplanatum — Stuxberg, 1876b: 317; Attems, 1904: 49; Lokšina, Golovatch, 1979: 383; Nefediev, Nefedieva, 2008a: 117.

Altajosoma pinetorum Gulička, 1972: 37, figs.

Altajosoma pinetorum — Lokšina, Golovatch, 1979: 382; Shelley et al., 2000: 62; Nefediev, Nefedieva, 2008a: 117.

Diplomaragna deplanata — Shear, 1990: 19, 20: figs; Mikhaljova, 1993: 22.

Diplomaragna pinetorum — Shear, 1990: 38; Mikhaljova, 1993: 25.

Altajosoma deplanatum - Mikhaljova, 2000: 160, figs; 2004 170, 171: figs, 162: map; 2013: 7; 2016: 12; 2017: 207, figs, 199: map; Mikhaljova, Golovatch, 2001: 108; Nefediev, 2002a: 30; 2002b: 35; Mikhaljova, Nefediev, 2003: 86; Nefediev, Nefedieva, 2005: 177; 2006: 98; 2007a: 139; 2007b: 161; 2007c: 99; 2008 62; 2011: 100; 2012a: 47; 2012b: 51; 2013: 87; 2017: 294; Mikhaljova et al., 2008: 51; Nefedieva, Nefediev, 2008: 123; Nefedieva et al., 2014: 65; 2015: 148.

MATERIAL EXAMINED. 2 9ᄋ, 3 juv., 1 fragm., Russia, Tomsk Area, Tomsk District, near Zorkaltsevo, $56.521326^{\circ} \mathrm{N}, 84$ $733903^{\circ} \mathrm{E}$, Pinus sibirica forest, p.t., 13-26.VI.2001; 4 우, s.1., p.t., 26.VI.-7.VII.2001; $2 \sigma^{7} \sigma^{7}, 6$ 우, 3 fragm., s.1., p.t., 724.VII.2001; 4 juv., s.1., soil samples, 24.VII. 2001; 7 ठ ठ $\sigma^{7}, 26$ 우, 8 fragm., s.1., p.t., 24.VII.-8.VIII.2001; $8 \sigma^{\top} \sigma^{\top}, 20$ 우, 3 fragm., s.1., p.t., 8-24.VIII.2001; 1 o', 1 juv., s.1., soil samples, 24.VIII.2001; $23 \Im^{7} \sigma^{7}, 33$ oᄋ, 4 juv., 2 fragm., s.l., p.t., 24.VIII.-20.IX.2001; 1 juv., s.1., 16.X.2001; 8 juv., same Area and District, near Kislovka, $56.430654^{\circ} \mathrm{N}, 84.900971^{\circ} \mathrm{E}$, Populus tremula forest, soil samples, 10-11.VI.2001; 3 juv., s.1., p.t., 10-26.VI.2001; 2 fragm., s.1., p.t.,

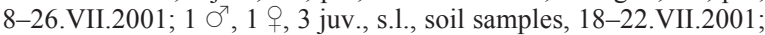

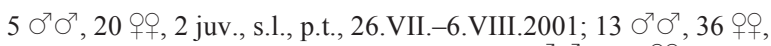
1 juv., 3 fragm., s.1., p.t., 6-26.VIII.2001; 40 ○ ఠ , 159 우, 4 juv., s.1., p.t., 26.VIII.-18.IX.2001; $2 \sigma^{\top} \sigma^{\top}, 4$ कo, 10 juv., 2 fragm., s.1., soil samples, 9.X.2001; 1 juv., same Area and District, near Petrovskii Uchastok, $56.512449^{\circ} \mathrm{N}, 84.789453^{\circ} \mathrm{E}$, Populus tremula and Betula pendula patch, soil samples, 20-26.VI.2001; 1 ๙ , 2 + + , 10 juv., s.1., soil samples, 8-10.VII.2001; 10 우, s.1., p.t., 8-24.VIII. 2001; $26 \sigma^{7} \sigma^{7}, 22$ OO, s.1., p.t., 24.VIII.-20.IX.2001; 7 juv., 1 fragm., s.1., 16.X.2001; 1 fragm., same Area and District, near Timiryzevskoye, $56.480920^{\circ} \mathrm{N}, 84.872666^{\circ} \mathrm{E}$, Betula pendula forest, p.t., 3-14.VI.2001; 2 ++, s.1., p.t., 9-26.VII.2001; $7 \sigma^{7} \sigma^{7}, 21$ OP, 1 juv., s.1., p.t., 26.VII.-6.VIII.2001; $4 \bigcirc^{\top} \sigma^{7}, 7$ की, s.1., p.t., 626.VIII.2001; 1 \% , 3 कo , near s.1., Betula pendula forest and Pinus sylvestris forest ecotone, p.t., 6-26.VIII.2001; $10 \sigma^{\top} \sigma^{\top}, 19$ 우, 1 juv., s.1., p.t., 26.VIII.-18.IX.2001, all leg. P.S. Nefediev, J.S. Nefedieva; 1 \% , same Area, Krivosheino District, near Novyi Istanbul, $57.418825^{\circ} \mathrm{N}, 83.878022^{\circ} \mathrm{E}$, mixed forest, 21.IX.2003, leg. P.S. Nefediev; $1 \sigma^{7}, 4$ 우, same Area, Tomsk District, near Zorkaltsevo, Pinus sibirica forest, 12.VII.-24.VIII.2006, leg. S.A. Krivets; $1 \sigma^{7}, 2$, 2 , Russia, Republic of Altai, Turochak District, Altai State Nature Reserve, NW of Lake Teletskoye, cordon Baigazan, $51^{\circ} 45^{\prime} 34.9^{\prime \prime} \mathrm{N}, 87^{\circ} 25^{\prime} 55.9^{\prime \prime} \mathrm{E}$, Padus avium forest with Sorbus sibirica, $460 \mathrm{~m}$ a.s.1., 30.IX.2013; $4 \Im^{\top} \sigma^{\top}, 1$ 9, near s.1., 51 $45^{\prime} 33.7^{\prime \prime} \mathrm{N}, 87^{\circ} 25^{\prime} 58.9^{\prime \prime} \mathrm{E}$, Padus avium forest with Sorbus sibiri$c a$, ca $470 \mathrm{~m}$ a.s.1., 30.IX.2013; $1 \mathrm{\sigma}^{7}, 3$ 우, near s.1., $51^{\circ} 45^{\prime} 35.0^{\prime \prime} \mathrm{N}$, $87^{\circ} 26^{\prime} 02.3^{\prime \prime} \mathrm{E}$, Salix caprea forest, ca $480 \mathrm{~m}$ a.s.1., 30.IX.2013; 2 $\sigma^{7} \sigma^{\prime}$, near s.1., $51^{\circ} 45^{\prime} 32.1^{\prime \prime} \mathrm{N}, 87^{\circ} 25^{\prime} 54.8^{\prime \prime} \mathrm{E}$, Padus avium forest, ca $455 \mathrm{~m}$ a.s.1., 1.X.2013; $1 \mathrm{O}^{\mathrm{T}}, 6$ 6 , , near s.1., $51^{\circ} 45^{\prime} 34.1^{\prime \prime} \mathrm{N}, 87^{\circ}$ 26'03.6"E, Padus avium forest, $490 \mathrm{~m}$ a.s.1., 1.X.2013; $1 \sigma^{7}$, near s.1., $51^{\circ} 45^{\prime} 35.0^{\prime \prime} \mathrm{N}, 87^{\circ} 25^{\prime} 42.2^{\prime \prime} \mathrm{E}$, Padus avium forest, ca $470 \mathrm{~m}$ a.s.1., 3.X.2013; $2 \sigma^{\top} \sigma^{7}, 1$, 1 , near s.1., $51^{\circ} 45^{\prime} 31.6^{\prime \prime} \mathrm{N}, 87^{\circ} 25^{\prime} 39.1^{\prime \prime} \mathrm{E}$, Padus avium, Salix caprea and Betula pendula forest, ca $450 \mathrm{~m}$ a.s.1., 3.X.2013, all leg. M.B. Sakhnevich; $1 \sigma^{7}, 1$ \%, Russia, Altai Province, Zmeinogorsk District, $3 \mathrm{~km} \mathrm{~N}$ of Zmeinogorsk, 51. $207239^{\circ} \mathrm{N}, 82.170883^{\circ} \mathrm{E}$, meadow, $400 \mathrm{~m}$ a.s.1., 29.VI.2016, leg.

Fig. 3. Distribution of Altajosoma species: corniferum Mikhaljova, Nefediev et Nefedieva, 2008 (square) and deplanatum (Stuxberg, 1876) (circle). Previously known localities marked in black, new records given in white.

Рис. 3. Распространение видов Altajosoma: corniferum Mikhaljova, Nefediev et Nefedieva, 2008 (квадрат) и deplanatum (Stuxberg, 1876) (круг). Черным отмечены ранее известные места находок, новые находки отмечены белым. 
Yu.V. Dyachkov; $1 \sigma^{7}$, Russia, Kemerovo Area, Krapivinskii District, 5-6 km of Taradanovo, 54 $44^{\prime} \mathrm{N}$, 86 $41^{\circ} \mathrm{E}$, Populus tremula forest, $300 \mathrm{~m}$ a.s.1., in litter and rotten logs, 13.VIII.2017; $1 \sigma^{7}$, 1 , same Area, Izhmorskii District, near Simbirka, $56^{\circ} 02^{\prime} \mathrm{N}$, $87^{\circ} 10^{\prime} \mathrm{E}$, Pinus sylvestris forest, $175 \mathrm{~m}$ a.s.1., in litter, 18.08.2018, all leg. D.A. Efimov.

DISTRIBUTION. This species is rather widespread in SW Siberia: S districts of the Tomsk Area, the Kemerovo Area as a whole, NW districts of the Republic of Khakassia, E districts of the Novosibirsk Area, $\mathrm{N}$ and $\mathrm{S}$ districts of the Altai Province, $\mathrm{N}$ and central districts of the Republic of Altai (Fig. 3).

REMARKS. The above new records show the NW and SW range limits of the species' distribution. An examination of males of the species from the collection of ASU has revealed a small anterior process (ap) on each angiocoxite of the posterior gonopods. This character is well visible both in fig. 32 of the redescription (see Shear [1990]) and a SEM micrograph (Figs 4, 5), but it was specified neither in that redescription nor later (see Mikhaljova [2017]). Hence, this structure must be included into the description of $A$. deplanatum.

\section{Altajosoma katunicum Mikhaljova, 2000}

\section{Fig. 1.}

Altajosoma katunicum Mikhaljova, 2000: 161, 162: figs.

Altajosoma katunicum - Mikhaljova, Golovatch, 2001: 108; Mikhaljova, 2004: 176, 177: figs, 112: map; Nefediev, Nefedieva, 2007b: 162; 2008: 62 .

non Altajosoma katunicum - Mikhaljova et al., 2008: 52; Nefedieva, Nefediev, 2008: 123; Nefediev, Nefedieva, 2013: 87; Nefedieva et al., 2014: 65; 2015: 149.

MATERIAL EXAMINED. $1 \sigma^{7}$, Russia, Republic of Altai, Ust-Koksa District, 25 air-km SE of Multa, valley of Multa River, $49^{\circ} 56^{\prime} 45.88^{\prime \prime} \mathrm{N}, 85^{\circ} 51^{\prime} 05.09^{\prime \prime} \mathrm{E}$, Pinus sibirica and Larix sibirica forest, $1700 \mathrm{~m}$ a.s.1., 18.VIII.2016; $1 \sigma^{7}, 1$, same Republic and District, 7 air-km SE of Multa, valley of Multa River, $50^{\circ} 06^{\prime} 03$ $63^{\prime \prime} \mathrm{N}, 85^{\circ} 54^{\prime} 45.56^{\prime \prime} \mathrm{E}$, Pinus sibirica and Betula pendula forest, $1200 \mathrm{~m}$ a.s.1., 23.VIII.2016, all leg. Yu.V. Dyachkov.

DISTRIBUTION. The distribution area of this species is restricted by the Katunskii Mt. Range in the Ust-Koksa District, Republic of Altai (Fig. 1).

REMARKS. The records of A. katunicum in the Altai State Nature Reserve, Ulagan District, Republic of Altai (see Mikhaljova et al. [2008]) turned out to be misidentifications, as all of them actually belong to A. baltyrgan and $A$. kemerovo.

\section{Altajosoma kemerovo (Shear, 1990)}

Fig. 2.

Diplomaragna kemerovo Shear, 1990: 21, 20: figs.

Diplomaragna kemerovo - Mikhaljova, 1993: 25; Nefediev, Nefedieva, 2008a: 117.

Altajosoma kemerovo - Mikhaljova, 2000: 161; 2004: 180, figs, 173: map; 2013: 7; Mikhaljova, Golovatch, 2001: 108; Vorobiova et al., 2002: 60; Mikhaljova, Nefediev, 2003: 86; Nefediev, Nefedieva, 2006: 98; 2007a: 139; 2007b: 161; 2008: 62; 2013: 87; Nefedieva, Nefediev, 2008: 123; Mikhaljova et al., 2008: 52; Nefedieva et al., 2014: 65; 2015: 149.

Altajosoma katunicum pro parte - Mikhaljova et al., 2008: 52; Nefedieva, Nefediev, 2008: 123; Nefediev, Nefedieva, 2013: 87; Nefedieva et al., 2014: 65; 2015: 149.

NEW MATERIAL EXAMINED. $1 \sigma^{\prime}, 2$, 9 , Russia, Republic of Altai, Turochak District, Altai State Nature Reserve, NW of Lake Teletskoye, cordon Baigazan, $51^{\circ} 45^{\prime} 32.7^{\prime \prime} \mathrm{N}, 87^{\circ} 26^{\prime} 05.8^{\prime \prime} \mathrm{E}$, Padus avium and Betula pendula forest, ca $480 \mathrm{~m}$ a.s.1., 1.X.2013; $1 \sigma^{\prime \prime}$, near s.1., $51^{\circ} 45^{\prime} 35.0^{\prime \prime} \mathrm{N}, 87^{\circ} 25^{\prime} 42.2^{\prime \prime} \mathrm{E}$, Padus avium forest, ca $470 \mathrm{~m}$ a.s.1., 3.X.2013, all leg. M.B. Sakhnevich.
MATERIAL RE-EXAMINED (specimens previously identified as A. katunicum and published by Mikhaljova et al. [2008]). 1 $\checkmark$, Russia, Republic of Altai, Ulagan District, Altai State Nature Reserve, S of Lake Teletskoye, cordon Chiri, mouth of Chiri River, $51.363121^{\circ} \mathrm{N}, 87.835473^{\circ} \mathrm{E}$, Betula pendula forest, $465 \mathrm{~m}$ a.s.1., 11-12.VIII.2005; 1 ○', same Republic, same District, Altai State Nature Reserve, S of Lake Teletskoye, near cordon Chiri, watershed of Kyga River and Bayas River, Kyga Biocenoses Profile, Site $1,51^{\circ} 20^{\prime} 47.3^{\prime \prime} \mathrm{N}, 87^{\circ} 51^{\prime} 14.2^{\prime \prime} \mathrm{E}$, Pinus sylvestris and Betula pendula forest, ca $445 \mathrm{~m}$ a.s.1., 12.VIII.2005, all leg. P.S. Nefediev, J.S. Nefedieva.

DISTRIBUTION. This species is rather widespread in SW and central Siberia: W districts of the Kemerovo Area, E districts of the Novosibirsk Area, W districts of the Republic of Khakassia, $\mathrm{N}$ districts of the Republic of Altai, as well as $\mathrm{S}$ districts of the Krasnoyarsk Province down the Yenisei River to $60^{\circ} 26^{\prime} \mathrm{N}$ (Fig. 2).

REMARKS. A restudy of two $\sigma^{\top} \sigma^{\top}$ from near cordon Chiri in the environs of Lake Teletskoye, both previously identified as A. katunicum by Mikhaljova et al. [2008], shows that they actually belong to A. kemerovo.

\section{Conclusions}

A re-examination of the type material of both subspecies of Altajosoma bakurovi (Shear, 1990) shows that: (1) A. bakurovi has no subspecific taxa and its full species status is revalidated; (2) the subspecies $A . b$. longiprocessum Mikhaljova, 2000 is elevated to a full species rank.

New records refine the distributions of seven Altajosoma species. Altajosoma bakurovi (Shear, 1990) is new to the Republic of Khakassia. Two species are recorded from outside their type localities for the first time: A. baltyrgan Mikhaljova, 2013 is found in the environs of Lake Teletskoye, while A. corniferum Mikhaljova, Nefediev et Nefedieva, 2008 is reported from the Altai Province. The NW and SW range limits of $A$. deplanatum (Stuxberg, 1876) are expanded, as opposed to A. katunicum Mikhaljova, 2000, which distribution area is reduced to the Katunskii Mt. Range. The presence of a small anterior process (ap) on each angiocoxite of the posterior gonopods of $A$. deplanatum must supplement the description of that species, as well as the diagnosis of the genus in general, i.e. anterior angiocoxal processes of the posterior gonopods are always present.

Acknowledgements. I am very thankful to S.I. Golovatch (Moscow, Russia) who kindly checked the English of an advanced draft. I am very grateful to all curators and colleagues from several academic institutions in Russia for the access to the diplopod collections under their care: S.I. Golovatch (ZMUM), R.V. Yakovlev (ZMUM and FSCB), A.A. Schileyko, E.N. Kudryavtseva (both ZMUM), E.V. Mikhaljova and A.S. Lelej (both FSCB). My deepest gratitude is extended to all persons who provided material for the present study: S.V. Dragan (Abakan, Russia), Yu.V. Dyachkov (Barnaul, Russia), D.A. Efimov (Kemerovo, Russia), A.A. Fomichev (Novosibirsk, Russia), V.I. Gusarov (Oslo, Norway), S.A. Krivets (Tomsk, Russia), T.M. Krugova (Barnaul, Russia), and M.B. Sakhnevich (Baigazan, Russia). I am very much obliged to R.Yu. Dudko (ISEA) who kindly provided the facilities for taking SEM micrographs and assisted in using them. 


\section{References}

Attems C. 1904. Myriopoden // Fauna Arctica. Bd.3. S.33-54.

Gulička J. 1972. [New millipedes (Diplopoda) from the USSR Part 2] // Zoologicheskii zhurnal. Vol.51. No.1. P.36-45 [in Russian, with English summary].

Lokšina I.E., Golovatch S.I. 1979. Diplopoda of the USSR fauna // Pedobiologia. Bd.19. S.381-389.

Mikhaljova E.V. 1993. The millipedes (Diplopoda) of Siberia and the Far East of Russia // Arthropoda Selecta. Vol.2. No.2. P.3-36.

Mikhaljova E.V. 2000 [for 1999]. Review of the millipede family Diplomaragnidae (Diplopoda: Chordeumatida) // Arthropoda Selecta. Vol.8. No.3. P.153-181.

Mikhaljova E.V. 2004. The millipedes (Diplopoda) of the Asian part of Russia. Sofia-Moscow: Pensoft Publishers. Series Faunistica 39. $292 \mathrm{p}$.

Mikhaljova E.V. 2013. New data on the millipede fauna (Diplopoda) of Altai, Russia // Far Eastern Entomologist. No.265. P.1-10.

Mikhaljova E.V. 2016. New species and new records of millipedes (Diplopoda) from the Asian part of Russia // Far Eastern Entomologist. No.316. P.1-25.

Mikhaljova E.V. 2017. [The millipede fauna (Diplopoda) of the Asian part of Russia]. Vladivostok: Dalnauka Publ. 336 p. [in Russian, with English summary].

Mikhaljova E.V. 2019. Millipedes of the order Chordeumatida (Diplopoda) from the Russian Far East and Siberia in the collection of the Natural History Museum of Denmark // Far Eastern Entomologist. No.374. P.1-9.

Mikhaljova E.V., Golovatch S.I. 2001 [for 2000]. A review of the millipede fauna of Siberia (Diplopoda) // Arthropoda Selecta. Vol.9. No.2. P.103-118

Mikhaljova E.V., Nefediev P.S. 2003 [for 2002]. A contribution to the millipede fauna of Siberia (Diplopoda) // Arthropoda Selecta. Vol.11. No.1. P.81-87.

Mikhaljova E.V., Nefediev P.S., Nefedieva J.S. 2008. A new species and new records of millipedes of the family Diplomaragnidae (Diplopoda, Chordeumatida) from Altai // Zootaxa. Vol.1931. P.49-56.

Nefediev P.S. 2002a. On the Diplopoda fauna of the South-West Siberia // Abstracts of 12th International Congress of Myriapodology. 28 July-3 August 2002, Mtunzini, KwaZulu-Natal, South Africa. P.30.

Nefediev P.S. 2002b. [The fauna and ecology of myriapods (Myriapoda) of a relict lime grove (village of Kuzedeyevo)] // Student $\mathrm{i}$ nauchno-tekhnicheskii progress: Biologiya. Materialy XL Mezhdunarodnoi nauchnoi studencheskoi konferentsii. Novosibirsk: Novosibirsk State University Publ. P.35 [in Russian].

Nefediev P.S., Nefedieva J.S. 2005. [Seasonal fluctuations of diplopod density in some forests of Western Siberia] // B.R. Striganova (ed.) Ekologicheskoe raznoobrazie pochvennoi bioty biologicheskaya produktivnost pochv. Materialy dokladov IV (XIV) Vserossiiskoi konferentsii po pochvennoi zoologii. 1-4 February 2005, Tyumen. Tyumen: Tyumen State University Publ. P.177-178 [in Russian].

Nefediev P.S., Nefedieva J.S. 2006. [Regional peculiarities of millipede fauna (Diplopoda) in the south-east of Western Siberia] // V.V. Anyushkin (ed.) Ekologiya Yuzhnoi Sibiri i sopredelnykh territorii. Sbornik materialov X Mezhdenarodnoi shkolykonferentsii studentov i molodykh uchionykh. Abakan: Khakassian State University Publ. Vol.10. No.1. P.98 [in Russian].

Nefediev P.S., Nefedieva J.S. 2007a. A brief analysis of the biotopic distribution of millipedes (Diplopoda) in the south-east of Western Siberia // A.I. Taskaev (ed.) Lesnoye pochvovedenie: itogi, problem, perspektivy. Tezisy dokladov Mezhdunarodnoi nauchnoi konferentsii. 4-11 September 2007, Syktyvkar. Syktyvkar: Institute of Biology, Komi Scientific Center, Ural Division of RAS Publ. P.139-140.

Nefediev P.S., Nefedieva J.S. 2007b. [Biogeographical characteristic of the millipede fauna in the southeast of Western Siberia // G.P. Ostroverkhova (ed.) Bioraznoobrazie bespozvonochnykh zhivotnykh. Sbornik materialov II Vserossiiskoi shkolyseminara s mezhdunarodnym uchastiem. 24-26 October 2007, Tomsk. Tomsk: Deltaplan Publ. P.159-164 [in Russian].

Nefediev P.S., Nefedieva J.S. 2007c. [Seasonal dynamics of locomotor activity of millipedes (Diplopoda) in forests of Western Siberia] // V.N. Kazin (ed.) Ekologicheskie problemy unikalnykh prirodnykh i antropogennykh landshaftov. Sbornik materialov Vserossiiskoi nauchno-prakticheskoi konferentsii. 29 November 2007, Yaroslavl. Yaroslavl: Yaroslavl State Univer- sity Publ. P.98-103 [in Russian].

Nefediev P.S., Nefedieva J.S. 2008a. [A historical review of faunistic investigations of millipedes (Diplopoda) in western Siberia] // V.M. Vazhov (ed.) Altai: ekologiya i prirodopolzovanie. Trudy VII rossiisko-mongolskoi nauchnoi konferentsii molodykh uchionykh i studentov. Vol.1. Biysk: Biysk Pedagogical State University Publ. P.117-120 [in Russian].

Nefediev P.S., Nefedieva J.S. 2008b. Zoogeographical analysis of the millipede fauna (Diplopoda) in the south-east of Western Siberia // Myriapoda and Onychophora of the World Diversity, Biology and Importance. Abstracts of 14th International Congress of Myriapodology. Staatliches Museum für Naturkunde, Görlitz. Peckiana. Vol.6. P.62.

Nefediev P.S., Nefedieva J.S. 2011. [Millipedes (Diplopoda) of green plantations of the city of Tomsk and its suburbs] // Kontseptualnye i prikladnye aspekty nauchnykh issledovanii $\mathrm{v}$ oblasti zoologii bespozvonochnykh. Sbornik materialov III Vserossiiskoi shkoly-seminara s mezhdunarodnym uchastiem, posvyaschionnoi 120-letiyu so dnya rozhdeniya Rostislava Petrovicha Berezhkova (1891-1961). 24-27 October 2011, Tomsk. Tomsk: Agraf-Press Publ. P.100-102 [in Russian].

Nefediev P.S., Nefedieva J.S. 2012a. [Some peculiarities of the distribution of millipedes (Diplopoda) along a soil profile in the south-taiga and small-leaved forests of Western Siberia // Izvestiya Altaiskogo gosudarstvennogo universiteta, Biologicheskie nauki. Vol.3(75). No.1. P.49-54 [in Russian, with English summary].

Nefediev P.S., Nefedieva J.S. 2012b. [Some peculiarities of the seasonal dynamics of sex-age structure in millipede populations (Diplopoda) in the southern taiga and small-leaved forests of Western Siberia // Izvestiya Altaiskogo gosudarstvennogo universiteta, Biologicheskie nauki. Vol.3(75). No.2. P.4648 [in Russian, with English summary].

Nefediev P.S., Nefedieva J.S. 2013. [Biodiversity and ecology of millipedes in the environs of Lake Teletskoye (Diplopoda)] // Izvestiya Altaiskogo gosudarstvennogo universiteta, Biologicheskie nauki. Vol.3(79). No.1. P.86-87 [in Russian, with English summary].

Nefediev P.S., Nefedieva J.S. 2017. New data on the millipede fauna of the Russian Altais, southwestern Siberia (Diplopoda)// Arthropoda Selecta. Vol.26. No.4. P.288-296.

Nefedieva J.S., Nefediev P.S. 2008. Ecofaunistical investigations of millipedes (Diplopoda) in the environs of Lake Teletskoe // Myriapoda and Onychophora of the World Diversity, Biology and Importance. Abstracts of 14th International Congress of Myriapodology. Staatliches Museum für Naturkunde, Görlitz. Peckiana. Vol.6. P.123-124.

Nefedieva J.S., Nefediev P.S., Sakhnevich M.B., Dyachkov Yu.V. 2014. Distribution of millipedes (Diplopoda) along an altitudinal gradient in the south of Lake Teletskoye, Altai Mts // Tuf I.H., Tajovský K. (eds.). 16th International Congress of Myriapodology. Book of abstracts. Institute of Soil Biology, BC ASCR \& Faculty of Science, Palacký University, Olomouc. P.65.

Nefedieva J.S., Nefediev P.S., Sakhnevich M.B, Dyachkov Yu.V. 2015. Distribution of millipedes (Diplopoda) along an altitudinal gradient in the south of Lake Teletskoye, Altai Mts, Russia // ZooKeys. Vol.510. P.141-161.

Shear W.A. 1990. On the Central and East Asian milliped family Diplomaragnidae (Diplopoda, Chordeumatida, Diplomaragnoidea) // American Museum Novitates. No.2977 P.1-40.

Shelley R.M., Sierwald P., Kiser S.B., Golovatch S.I. 2000. Nomenclator generum et familiarum Diplopodorum II. A list of the genus and family-group names in the class Diplopoda from 1958 through 1999. Sofia-Moscow: Pensoft Publishers. $167 \mathrm{p}$.

Stuxberg A. 1876a. Myriopoder från Sibirien och Waigatsch ön samlade under Nordenskiöldska expeditionen 1875 // Öfversigt af Kongliga Vetenskaps-Akademiens Förhandlingar. Årg.33. No.2. S.11-38

Stuxberg A. 1876b. On the Myriopoda, from Siberia and Waigatsch Island, collected during the expedition of Prof. Nordenskiöld, 1875 // Annals and Magazine of Natural History. Vol.4. No.17. P.306-318. doi: 10.1080/00222937608681955

Vorobiova I.G., Rybalov L.B., Rossolimo T.E., Zalesskaja N.T. 2002. [Zonal and landscape distribution of the myriapod fauna and populations (Myriapoda) in the Yenisei River basin] // Izuchenie, sokhranenie i vosstanovlenie bioraznoobraziya ekosistem na Yeniseiskom ekologicheskom transekte: Zhivotnyi mir, etno-ekologicheskie issledovniya, 2. Moscow: IEE RAS Publ. P.60-71 [in Russian]

Responsible editor S.I. Golovatch 